PRECISE ATOMIC DATA

\author{
Martin C.E. Huber* \\ ESA/ESTEC, Space Science Department \\ Postbus 299, 2200 AG Noordwijk \\ The Netherlands
}

\title{
ABSTRACT
}

We discuss the three questions: Why do we need accurate atomic data for stellar spectroscopy? What accuracy is needed? And: How can the accuracy of oscillator strengths be assessed? In conclusion we comment on the state of the art, stress the importance of uncertainty estimates and also discern between precision and accuracy.

1. INTRODUCTION: WHY DO WE NEED ACCURATE DATA FOR STELLAR SPECTROSCOPY?

Abundance determinations require accurate atomic data for two interdependent reasons: (i) to derive a reliable abundance value from observed equivalent widths with the aid of a realistic atmosphere model and (ii) to check on the validity of this model - in particular, with regard to the actual (inhomogeneous) structure of the atmosphere and deviations from local thermodynamic equilibrium (LTE). Advances in both, instrumentation and interpretation have made it possible now to extend these latter tests from solar to stellar spectroscopy.

In instrumentation, the replacement of photographic film and plates by (imaging) photoelectric detectors (see, e.g., Timothy 1983) has resulted in an increase in signal-to-noise ratio - and thus also attainable resolving power - as well as in radiometric accuracy. Therefore, measurements of equivalent widths and line profiles are now limited in accuracy mostly by the uncertainty in the continuum position and extended instrumental line wings (Griffin 1969): currently, equivalent widths in high-resolution stellar spectra can probably be measured with an accuracy of three to four per cent. And in the solar case, an isolated, unblended line can probably be measured with an accuracy of two per cent. Here, double-pass grating spectrometers (Delbouille, Roland and Neven, 1973) or Fourier-transform spectrometers (FTS) (Kurucz et al., 1984) are now used and this results in instrumental profiles

* On leave of absence from Swiss Federal Institute of Technology Zurich (ETHZ), Institute of Astronomy.

G. Cayrel de Strobel and M. Spite (eds.), The Impact of Very High S/N Spectroscopy on Stellar Physics, 361-366. (c) 1988 by the IAU. 
with negligible wing intensities.

As far as interpretation is concerned, the construction of theoretical LTE model atmospheres for specific parameters, $\mathrm{T}_{e}, \log \mathrm{g}$ and metallicity is now almost routine. The MARCS program of Gustafsson et al. (1975), for example, is implemented on the UK STARLINK network. Nevertheless, Rutten and Kostik (1988) have shown, that LTE models lead to solar oscillator strengths for Fe $I$, that can be in error by 0.1 dex (where 0.1 dex is a logarithmic interval corresponding to a factor of $10^{0} 1$ ). Solanki and Steenbock (1987) also demonstrated that the use of homogeneous LTE models result in apparent abundance values that differ by a similar factor from those which consider non-LTE (NLTE) effects, and that even larger discrepancies must be expected, if parts of the inhomogeneous atmosphere (as, e.g., the chromospheric network) are considered separately. (These discrepancies are most pronounced for low-excitation lines of $\mathrm{Fe} I\left(\mathrm{E}_{1} 3.5 \mathrm{eV}\right)$, they are somewhat less important for higher excitation potentials and practically absent for Fe II.) NLTE atmospheres are now becoming more common and can be expected to be much developed in future.

\section{WHAT ACCURACY IS NEEDED?}

Oscillator strengths are not the only atomic data needed in interpreting solar and stellar spectra. As soon as a line is no more optically thin, damping and - in many cases - hyperfine splitting become of importance. Accuracy requirements for each of these quantities are treated in the following subsections.

\subsection{Atomic oscillator strengths}

To interpret properly the double-pass and FTS solar spectra, for which equivalent widths can be measured to an accuracy of two per cent, an accuracy in gf of one per cent or better is desirable. This accuracy is needed to test both, the extent of the applicability of LTE modelatmospheres and the improvements offered by NLTE models.

\subsection{Collision damping}

The effect of uncertainty in damping is usually underestimated and often completely overlooked (Blackwell, Calamai and Willis, 1972). As an example, a solar line with an equivalent width of $8 \mathrm{pm}$, a lower excitation potential of $4 \mathrm{eV}$ and a wavelength of $600 \mathrm{~mm}$, whose damping constant is in error by a factor of three will lead to an error of nearly 30 per cent in abundance. Although the error decreases quite rapidly with decreasing excitation and equivalent width, it is clear that, if equivalent widths of accuracy four per cent are to be interpreted using oscillator strengths of accuracy one per cent, great attention should be paid to the accuracy of the damping constants.

The rôle of damping in the solar spectrum is treated by Simmons and Blackwell (1982) and by Blackwell, Booth and Petford (1984a). The Fe-I 
damping constants used are those measured by $O^{\prime}$ Neill and Smith (1980a and 1980b). Further work referring to damping in the solar spectrum is reported by Blackwell et al. (1987). Calculations of sufficiently accurate damping constants for complex atoms will probably remain a very difficult undertaking. Much more experimental work is therefore needed!

\subsection{Hyperfine structure}

Hyperfine structure is another important, much neglected topic. Hyperfine splitting can be caused by both, the isotope shift (in the case of a mixture of isotopes) and by the interaction of a (non-zero) nuclear spin, I, with the total angular momentum, J, of the electrons. This often results in a number of close-lying line components. Hyperfine splitting makes its influence felt in laboratory measurements as well as in solar and stellar spectra. Examples for the case of Mn I are given by Booth et al. (1983a and 1984a) as well as by Booth et al. (1984b and 1983b), respectively. In brief, profiles "diluted" by hyperfine splitting change the behaviour of the curve of growth.

Much more work is needed here as well, but, fortunately, measurements are straightforward. They are probably taken most easily and reliably by use of a hollow cathode (or other narrow-line source) and a FTS.

\section{HOW CAN THE ACCURACY OF OSCILLATOR STRENGTHS BE ASSESSED?}

Assessing the accuracy of oscillator strengths is difficult. The following two methods have been used most frequently: (i) intercomparing values measured by different groups and (if ever possible) by different techniques, and (ii) comparing solar abundances derived by use of lines from the same multiplet.

An intercomparison of oscillator strengths for low-excitation lines of $\mathrm{Fe}-\mathrm{I}$ is shown in Figure 1. The dynamic range of the comparison extending over six decades - is remarkable. However, the methods used to obtain the data shown in this figure (the absorption method by Blackwell et al. (1979) and a combination of anomalous-dispersion and

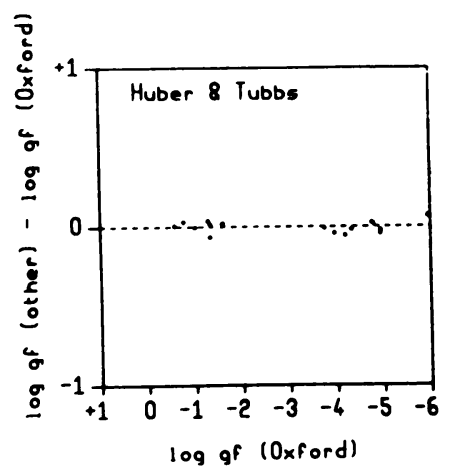

Figure 1.

Comparison of oscillator strengths for low-excitation lines of $\mathrm{Fe} I$ by Huber and Tubbs (1972) with those by Blackwell et al. (1979). 
absorption measurements by Huber and Tubbs (1972)) both depend on the population of the lower level of a given transition. A more thorough assessment can be made, if results from absorption and emission measurements are compared: the former depends on the population of the lower, and the latter on that of the upper levels of the lines in question. Tozzi, Brunner and Huber (1985) have compared their branching-fraction data for $\mathrm{Cr}$ I with the absorption data of Blackwell, Menon and Petford (1984b). To the best of our knowledge, this was the first comparison between laboratory transition probabilities where the claimed precision of the Oxford values could be confirmed - at least to the three-per-cent level. A formal procedure for such comparisons - now designated as the Ladenburg method (Huber and Sandeman 1986) - has been developed by Cardon, Smith and Whaling (1980).

If one compares derived solar abundances, the influence of NLTE effects can be minimised by exclusive use of lines belonging to one or several similar multiplets. The accuracy of such tests is somewhat degraded by the necessary solar equivalent-width measurements. Nevertheless, good results were obtained by Blackwell et al. (1987): sixteen Ti-I lines of excitation potential $1 \mathrm{eV}$, for example, resulted in an abundance value having a standard deviation of 3.4 per cent (for the most suitable microturbulent velocity of $0.85 \mathrm{~km} \mathrm{~s}^{-1}$ ).

\section{CONCLUSIONS AND OUTLOOK}

We are now entering a realm, where transition probabilities with a 10 per-cent precision are no longer sufficient. And, indeed, a precision of a few per cent for measured oscillator strengths of complex spectra has now been confirmed. Once this precision is reached, one must also consider (and measure!) damping coefficients and the hyperfine splitting.

Above all, however, astronomers and laboratory physicists must estimate uncertainties carefully; for, strictly speaking, a value without an uncertainty is meaningless. While it is necessary to have at least one accurate oscillator strength to obtain a reliable abundance value, precise (i.e. self-consistent, but possibly off-the-mark) data will do for tests of atmospheric models.

It should also be noted, that abundance determinations converge to a constant and (one would hope) correct value as the excitation potential increases (cf., e.g., Blackwell et al., 1987). For accurate abundance work it is, therefore, important to measure transition probabilities for high excitation lines.

\section{ACKNOWLEDGEMENT}

Prof. D.E. Blackwell's very kind help in preparing this paper is greatly appreciated. 


\section{REFERENCES}

Blackwell, D.E., Ibbetson, P.A., Petford, A.D. and Shallis, M.J., 1979, Mon. Not. R. astr. Soc., 186, 633.

Blackwell, D.E., Booth, A.J., Menon, S.L.R. and Petford, A.D., 1987, Astron. Astrophys. (in press) .

Blackwell, D.E., Calamai, G. and Willis, R.B., 1972, Mon. Not. R. astr. Soc., $160,121$.

Blackwell, D.E., Booth, A.J., Menon, S.L.R., Petford, A.D. and Smith, G., 1983, Mon. Not. R. astr. Soc., 204, 141.

Blackwell, D.E., Booth, A.J. and Petford, A.D., 1984a, Astron. Astrophys., 132, 236.

Blackwell, D.E., Menon, S.L.R. and Petford, A.D., 1984b, Mon. Not. R. astr. Soc., 207, 533.

Booth, A.J., Shallis, M.J. and Wells, M., 1983a, Mon. Not. R. astr. Soc., 205, 191.

Booth, A.J. and Blackwell, D.E., 1983b, Mon. Not. R. astr. Soc., 204, 777.

Booth, A.J., Blackwell, D.E., Petford, A.D. and Shallis, M.J., 1984a, Mon. Not. R. astr. Soc., 208, 147.

Booth, A.J., Blackwell, D.E. and Shallis, M.J., 1984b, Mon. Not. R. astr. Soc., 209, 77.

Cardon, B.L., smith, P.L. and Whaling, W., 1980, Phys. Rev. A, 20, 2411.

Delbouille, L., Roland, G. and Neven, L., 1973, Photometric Atlas of the Solar Spectrum from $3000 \AA$ to $10000 \AA$. Institut d'Astrophysique de l'Université de Liège, Belgium.

Griffin, R.F., 1969, Mon. Not. R. astr. Soc., 143, 319.

Gustafsson, B., Bell, R.A., Erikssen, K. and Nordlund, A., 1975, Astron. Astrophys., 42, 407.

Huber, M.C.E. and Tubbs, E.F. 1972., Astrophys. J., 177, 847.

Huber, M.C.E. and Sandeman, R.J., 1986, Rep. Prog. Phys. 49, 397.

Kurucz, R.L. and Peytremann, E., 1975, A table of semi-empirical gf-values, parts 1, 2, 3. Smithsonian Astrophys. Obs. Spec. Rep. 362.

Kurucz, R.L., Furenlid, I., Brault, J.W., Testerman, L., 1984, Solar Flux Atlas from 296 to $1300 \mathrm{~mm}$. National Solar Observatory Atlas No. 1.

O'Neill, J.A. and Smith, G., 1980a, Astron. Astrophys., 81, 100.

O'Neill, J.A. and Smith, G., 1980b, Astron. Astrophys., 81, 108.

Rutten, R.J. and Kostik, R.I., 1988, Physics of Formation of Fe II Lines Outside LTE, Proc. IAU Coll. No. 94, (Dordrecht: Reidel).

Simmons, G.J. and Blackwell, D.E., 1982, Astron. Astrophys., 112, 209.

Solanki, S.K. and Steenbock, W., 1987, Astron. Astrophys. (submitted).

Timothy, J.G., 1983, Publ. Astron. Soc. Pacific, 95, 810.

Tozzi, G.P., Brunner, A. and Huber, M.C.E., 1985, Mon. Not. R. astr. Soc., 217, 423.

Unsöld, A. 1955, Physik der Sternatmosphären, 2nd edn. (Berlin: Springer). 
DIscussion

GIRIDEAR Is there a correlation between the enhancenent factor to $C_{6}$ and excitation potential of the lines?

HUBER Yes, the enhancement factor tend to become higher for high excitation potential lines. (see, for example, simmons and Blackwe 11: 1982, Astron.Astrophys.112, 209)

MGAIN I wish to stress that the astrophysicists do not only need accurate oscillator strengths for high excitation neutral lines, but also for ion lines.

HUBER I agree ...

GRIFPIN You mentioned that the accuracy of stellar equivalent widths is in the best case about 3 or 4 percent. I should like to make the following cownent : two groups of astronomers, both claiming access to high $S / N$, high resolution observations of high accuracy, have published equivalent widths for 25 weak lines of $\mathrm{Fe} I$ in the same star. Their measurements differ by 20 percent.

CAYREL Is there any laboratory measurements planned for getting the broadening of neutral metallic lines by neutral hydrogen ?

HUBER Such measurements are very difficult, nevertheless some have already been made in the fifties (Kush H.J.:1958, Z. Ast rophys. 45, 1). Nodern measurements are made in Be and then corrected for the case of H by use of a semi-empirical theory (see, for example, O'Neill and smith :1980, Astron.Astrophys. 81, 108). 